\title{
Structure of Clean and Arsenic-covered GaN(0001) Surfaces
}

\author{
V. Ramachandran, C. D. Lee, and R. M. Feenstra \\ Department of Physics, Carnegie Mellon University, Pittsburgh, Pennsylvania \\ 15213 \\ A. R. Smith \\ Department of Physics and Astronomy, Ohio University, Athens, Ohio 45701 \\ J. E. Northrup \\ Xerox Palo Alto Research Center, 3333 Coyote Hill Road, Palo Alto, California \\ 94304 \\ D. W. Greve \\ Department of Electrical and Computer Engineering, Carnegie Mellon University, \\ Pittsburgh, Pennsylvania 15213
}

\begin{abstract}
The effect of trace arsenic on the growth and surface structure of $\mathrm{GaN}(0001)$ has been studied. We find that a partial pressure of only $10^{-9}$ Torr of arsenic during molecular beam epitaxial growth significantly modifies the growth kinetics. Such a small background pressure of arsenic leads to an arsenic-terminated surface displaying a $2 \times 2$ reconstruction $d u r-$ ing growth which is absent for the clean surface. First-principles theoretical calculations show that As-terminated surfaces are energetically more favorable than Ga-terminated surfaces for arsenic pressures of $10^{-9}$ Torr, and structural models for the As-adatom $2 \times 2$ reconstruction are presented.
\end{abstract}

\section{Introduction}

Despite remarkable progress in device development using $\mathrm{GaN}$ and related alloys over the past number of years, relatively few studies have been performed on the fundamental surface properties of the material. Studies of the atomic-scale structure of the surface are important from the point of view of understanding growth of the material. Also, as discussed below, observation of surface reconstructions can be used to determine the polarity of the material.[1] Polarity dependent etch rates of the material are determined by the detailed atomic arrangement of the surface,[2] and the surface geometry presumably affects many other processing steps used in device fabrication. Thus, it is important to determine the fundamental surface arrangements which occur on the surface.

Very early work on GaN surfaces using scanning tunneling microscopy (STM) was reported by Packard, Dow and co-workers. [3] More comprehensive work combining STM and first-principles theoretical computations was used by our group to identify the surface reconstructions for both the Ga-polar (0001) and the N-polar (0001) surfaces, prepared by molecular beam epitaxy (MBE).[4,5] Detailed work by Held et al. using thermal desorption spectroscopy has provided valuable information on the gallium coverage of GaN during growth.[6] Photoemission spectroscopy has revealed the presence of well defined dangling bond states on the surface, although the geometry (and polarity) of the surfaces studied there was not clear.[7,8] 
A major result of our prior work is that there exist two different families of reconstructions of GaN surfaces, one family associated with the (0001) surface and the other associated with the $(000 \overline{1})$ surface. Each surface can exist in a number of different structures depending on the surface stoichiometry (ranging from N-rich to Ga-rich). Figure 1 summarizes our results for these surface structures. We show a schematic phase diagram for the main surface reconstructions observed, together with the corresponding reflection high-energy electron diffraction (RHEED) patterns as viewed along the [11 $\overline{2} 0$ ] azimuth. For the $(000 \overline{1})$ surface, Fig. $1(\mathrm{a})$, a $1 \times 1$ structure is formed in the N-rich limit; this structure is produced by heating the as-grown film surface to high temperature $\left(\approx 800^{\circ} \mathrm{C}\right)$ in order to remove excess $\mathrm{Ga}$ adatoms. First-principles total energy calculations demonstrate that this $1 \times 1$ consists of a Ga monolayer (or adlayer) bonded to the uppermost N-terminated bilayer.[4] The $3 \times 3,6 \times 6$, and $c(6 \times 12)$ reconstructions are produced by depositing sub-monolayer quantities of $\mathrm{Ga}$ onto this $1 \times 1$ surface. These higher order reconstructions, however, only exist below $\approx 300^{\circ} \mathrm{C}$, above which they undergo reversible order-disorder phase transitions.[9] For the (0001) surface, Fig. 1(b), the most Ga-rich reconstruction is a " $1 \times 1$ " (pseudo- $1 \times 1$ ), consisting, we believe, of a double layer of $\mathrm{Ga}$ atoms in an incommensurate fluid-like state on top of the bulkterminated GaN bilayer.[10] Annealing this " $1 \times 1$ " followed by additional Ga deposition produces $5 \times 5$ and $6 \times 4$ reconstructions. Finally, in the N-rich limit achieved by interrupting the Ga supply during MBE growth, a $2 \times 2$ reconstruction can be observed which we associate with a $\mathrm{N}$-adatom structure [5].

Recently, some new STM results for the GaN(0001) surface have been published by Xue et al.[11] That group performed MBE growth of the GaN using an rf-plasma source for nitrogen, in a very similar way as done in our growth system. However, their results for the surface reconstructions are quite different. For the Ga-face, Xue et al. find a series of reconstructions including $2 \times 2,4 \times 4,5 \times 5,5 \sqrt{ } 3 \times 2 \sqrt{ } 13, \sqrt{ } 7 \times \sqrt{ } 7$, and $10 \times 10$, all of which differ from those reported in our work (differences between their $2 \times 2$ structure and that seen in our work are discussed below). In the Garich limit Xue et al. report 1×1-Ga-fluid structure, in apparent agreement with our Ga-rich result.

The $2 \times 2$ and $4 \times 4$ reconstructions reported by Xue et al. have been reported by many groups performing MBE growth of GaN.[12-14] The $2 \times 2$ in particular is seen by RHEED during growth and is often quite intense. These reconstructions have been used as indicators of both the Ga-polarity of the film and the high quality of the growth.[15] Nevertheless, a number of other groups, including our own, have been unable to observe a $2 \times 2$ RHEED pattern during growth (although all groups can achieve it when the growth is interrupted). We have previously discussed this apparent discrepancy between the results of the various groups, and have suggested that the $2 \times 2$ (and $4 \times 4$ ) may be the result of unintentional contamination in the vacuum chamber used for the growth.[14] Arsenic is a prime candidate for such contamination, since many of the growth chambers used for $\mathrm{GaN}$ have previously been used for GaAs growth, and the high vapor pressure of arsenic ensures that any trace amounts present near the growth sources will produce a significant partial pressure during growth. For our prior studies, neither the growth chamber nor any of its components had ever been exposed to any arsenic.

To test the sensitivity of the $2 \times 2$ reconstruction to the presence of arsenic, we have recently undertaken a set of measurements in which arsenic is purposely introduced into our growth chamber. In this paper we report on the results of these experiments. We find that under very Ga-rich conditions arsenic does not have any effect on the growth but under moderately Ga-rich conditions, 
it produces a $2 \times 2$ surface reconstruction both during growth and upon interrupting the growth. Furthermore, we find that depositing small amounts of Ga onto this $2 \times 2$ surface at temperatures around $250^{\circ} \mathrm{C}$ produces $4 \times 4$ and $5 \times 5$ reconstructions, in an analogous manner as that reported by Xue $e t$ al.[11] Associated with the occurrence of the $2 \times 2$ reconstruction, arsenic is also seen to prevent the roughening of films grown by MBE under mildly $\mathrm{N}$-rich conditions. This observation is in agreement with the results of other workers who find, in the presence of the $2 \times 2$ structure, a streaky RHEED pattern under N-rich conditions.[12,13] In contrast, in the absence of arsenic, the RHEED pattern is spotty (indicating rough growth) under N-rich conditions. [6,14,16]

\section{Experimental}

The GaN films investigated in these studies were grown by $\mathrm{MBE}$ on $\mathrm{Si}$-polar $6 \mathrm{H}-\mathrm{SiC}(0001)$ substrates, resulting in Ga-polar GaN films. MBE growth is performed at temperatures in the range $625-700^{\circ} \mathrm{C}$ using an rf-plasma $\mathrm{N}$ source and an effusion cell for Ga. Films are grown under Ga rich conditions, as described in more detail elsewhere.[17] An arsenic flux was produced by resistive heating of a GaAs wafer located in the growth chamber about $5 \mathrm{~cm}$ from the substrate holder. For about 2.4 A of current through the wafer an arsenic beam equivalent pressure (BEP) of $1 \times 10^{-9}$ Torr was observed. Monitoring of the surface during growth was performed by in situ RHEED. Experiments were performed by sweeping Ga flux at the surface during growth from $4.5 \times 10^{14} \mathrm{~cm}^{-2} \mathrm{~s}^{-1}$ down to $2.5 \times 10^{14} \mathrm{~cm}^{-2} \mathrm{~s}^{-1}$ with and without arsenic exposure and also by keeping Ga flux constant while turning on and off the arsenic source.

\section{Results and Discussion}

In the absence of any arsenic flux, it is known that during growth the GaN surface roughens when the $\mathrm{Ga}$ flux is decreased from a Ga-rich growth regime to a N-rich regime.[16] Theoretical models for this roughening transition state that under $\mathrm{N}$-rich conditions areas of the surface are covered with excess N.[18] When Ga atoms arrive at these areas, their mobility is severely reduced owing to the fact that $\mathrm{Ga}-\mathrm{N}$ bonds are formed very quickly, and this reduction in $\mathrm{Ga}$ atom mobility leads to three-dimensional growth.[18] In Fig. 2(a), we show the dim and streaky 1×1 RHEED pattern seen under our normal Ga-rich growth conditions. As the Ga flux is reduced the RHEED pattern brightens as shown in Fig. 2(b) and then becomes spotty (Fig. 2(c)) indicative of roughening. These measurements have been repeated while varying the current in the arsenic source. For arsenic BEP below $1 \times 10^{-9}$ Torr, no change is detected in the sequence mentioned above. However, for higher arsenic BEP, as the Ga flux is reduced, a bright streaky $2 \times 2$ RHEED pattern is observed as shown in Fig. 2(e).

In Fig. 3, we show a plot of our observations of the surface under different Ga and As flux conditions. The lines $\alpha, \beta$ and $\gamma$ indicate the different experiments we performed. As we decrease the gallium flux for fixed, low arsenic flux we see the direct transition from a streaky $1 \times 1$ to a spotty RHEED pattern (experiment $\alpha$ ). Above an arsenic BEP of about $1 \times 10^{-9}$ Torr we see an intermediate bright $2 \times 2$ phase appear as we reduce the Ga flux (experiment $\beta$ ). At sufficiently low Ga flux the RHEED pattern again becomes a spotty $1 \times 1$ (this transition to spotty $1 \times 1$ is somewhat gradual, presumably because of the same kinetic limitations which give rise to the spotty $1 \times 1$ RHEED pattern itself [18]). The line $\gamma$ shows experiments performed at fixed Ga flux while increasing the arsenic flux. For Ga cell temperatures in the range $1055-1065^{\circ} \mathrm{C}$ in Fig. 3, in the presence of As, there is a marked transition to/from the $2 \times 2$ phase. In Fig. 3(b), we show the intensity of the half order streaks in the RHEED pattern as a function of time for the three experiments $\alpha, \beta$ and $\gamma$, showing 
the appearance of these streaks upon exposure to arsenic. Clearly, the $2 \times 2$ persists well after the As flux is turned off (experiment $\gamma$ ), indicating that the As atoms segregate to the surface during the GaN growth. Having established a $2 \times 2$ reconstruction, we find that it remains unchanged through growth interruption and cooling down to room temperature.

Also seen in our phase diagram is the fact that in the presence of arsenic the roughening transition of the surface occurs at Ga fluxes smaller than those in the absence of arsenic. This indicates the possibility that arsenic may increase the mobility of $\mathrm{Ga}$ atoms impinging on the film in $\mathrm{N}$ covered areas, preventing roughening. This fact, coupled with the surface segregation mentioned above, strongly suggests that arsenic is behaving as a surfactant in GaN growth. This surfactant behavior of arsenic has previously been reported by Zhao et al., although their experiments used $\mathrm{AsH}_{3}$ for the arsenic source with a much higher flux than in our experiments [19]. In our case, for higher arsenic fluxes (near the top of the phase diagram of Fig. 3), it appeared that the presence of the arsenic roughened the GaN surface. In particular, as the Ga flux was reduced, the $2 \times 2$ appeared briefly, but throughout most of the Ga flux sweep the RHEED pattern was spotty.

Based on these observations we conclude that arsenic changes the surface structure during growth. Under highly Ga rich conditions, when the surface is covered with several $(\approx 2)$ disordered monolayers (ML) of Ga, arsenic atoms accumulate on the surfaces, presumably incorporating into the layers of $\mathrm{Ga}$ in a disordered fashion. However, as the $\mathrm{Ga}$ flux at the surface decreases and with sufficient arsenic coverage, it becomes energetically favorable for the surface to convert to an arsenic covered $2 \times 2$ structure. A simple model for this structure would consist of one As-adatom per $2 \times 2$ unit cell,[20] although as described below an As-trimer model is also possible. We have also observed that depositing small amounts of $\mathrm{Ga}$ on the $2 \times 2$ surface at temperatures near $250^{\circ} \mathrm{C}$ leads to the formation of $4 \times 4$ and $5 \times 5$ reconstructions, in agreement with the observations of Xue et al.[11] and thus providing additional evidence that the arsenic-induced $2 \times 2$ structure observed here is the same as that seen of Xue et al. A simple model for the $4 \times 4$ structure would consist of 3 arsenic adatoms and $1 \mathrm{Ga}$ adatom per $4 \times 4$ cell, yielding STM contrast consistent with that reported by Xue et al. (dangling bonds are filled on As adatoms and empty on Ga adatoms).

To further demonstrate the presence of arsenic in the $2 \times 2$ structure of the GaN(0001) face, we have performed Auger emission studies of this surface, as shown in Fig. 4. Auger emission lines from $\mathrm{Ga}$ and $\mathrm{N}$ are seen, together with some smaller lines arising from $\mathrm{C}$ and $\mathrm{O}$ surface contamination. The source of this $\mathrm{C}$ and $\mathrm{O}$ is believed to be outgassing from the electron gun in the Auger system itself, since we observe this contamination signal to increase due to exposure to the Auger system (also, in the absence of any Auger measurement our surfaces appear quite clean in STM images, whereas after Auger measurement the surfaces appear significantly contaminated in the STM images). Near $1230 \mathrm{eV}$ we clearly see a small Auger signal characteristic of arsenic. Based on our Auger evaluation parameters described elsewhere,[10] we find that the intensity of this signal corresponds to about $0.18 \mathrm{ML}$ coverage of arsenic at the surface. This value is somewhat less than the surface coverage of $0.25 \mathrm{ML}$ expected for the simple $2 \times 2$ As-adatom structure, but the difference is within the uncertainty of our Auger measurement. The coverage deduced from Auger is considerably less than that for an As-trimer model, discussed below, thus favoring the $2 \times 2$ As-adatom model as the actual structure of our surface.

\section{Theory}


The experimental results described above indicate that an As pressure greater than about $10^{-9}$ Torr is required to form the $2 \times 2$ structure when growth occurs at a sample temperature of $\mathrm{T}=700^{\circ} \mathrm{C}$. An important question is whether the theory of the relative energetics of the clean and As-adatom terminated (0001) surfaces is consistent with this fact. To answer this question, total energy calculations were performed for As-containing structures that could give rise to a $2 \times 2$ RHEED pattern. These calculations were performed using first-principles pseudopotentials, a plane wave basis set for the expansion of the wave functions, and a supercell approach. The maximum energy of plane wave basis set was $60 \mathrm{Ry}$, and the GaN $3 \mathrm{~d}$ electrons were included in the valence band. These techniques have been employed previously to study clean $\mathrm{GaN}$ surfaces [22] as well as adsorption of foreign species such as As [20,23] and $\mathrm{H}[24]$ on these surfaces. Previous calculations for As-adatoms on the $\mathrm{GaN}(0001)$ surface [20] indicate that the As-terminated surface is more stable than the Ga-terminated surface when the As is equilibrated with GaAs. We will show here that the $2 \times 2$ Asadatom structure is more stable than the Ga-terminated surfaces even when the As pressure is as low as $10^{-9}$ Torr.

Three types of structural models were considered: The $2 \times 2$ As-adatom models, with the adatoms occupying $\mathrm{H} 3$ or T4 sites (Figs. 5 and 6 respectively), and a $2 \times 2$ As-trimer model, with the trimer in the T4 site (Fig. 7). The atomic positions were determined by minimizing the total energy. In the As-adatom structure, each As-adatom forms bonds with three neighboring Ga atoms. The length of these bonds is calculated to be 2.52(2.54) $\AA$ for the H3(T4) structure. The vertical height of the adatom, relative to the underlying Ga layer, is 1.83(1.90) $\AA$ for these structures. The energies of the $\mathrm{H} 3$ and $\mathrm{T} 4 \mathrm{As}$ adatom structures differ by only $0.1 \mathrm{eV} /(2 \times 2)$ with the $\mathrm{H} 3$ structure being lower in energy. The atomic relaxation of the substrate $\mathrm{GaN}$ is quite extensive in both cases. In the As-trimer structure three As atoms are mutually bonded, forming an equilateral triangle with As-As bonds of length $2.48 \AA$. Each of these As atoms is bonded to a Ga atom below, and the length of the As-Ga bonds is $2.44 \AA$. The vertical height of the trimer is $2.42 \AA$ above the underlying Ga layer. For both adatom and trimer structures the 4th $\mathrm{Ga}$ atom in the cell in the surface layer (the restatom) adopts an $\mathrm{sp}^{2}$ configuration with an empty $\mathrm{Ga}_{z}$-orbital. Both adatom and trimer structures satisfy the electron counting rule.

The relative energies of the As-adatom, As-trimer, and the Ga-terminated GaN(0001) surfaces depend on the chemical potentials $(\mu A s, \mu G a)$ of the various species. In extreme As-rich con-

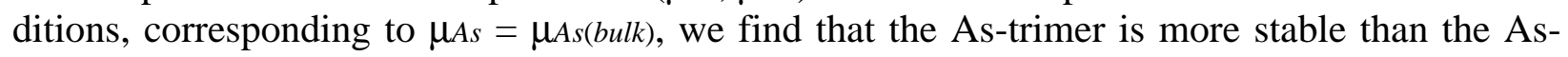
adatom by $0.98 \mathrm{eV} /(2 \times 2)$. Thus, when $\mu A s<\mu A s(b u l k)-0.49 \mathrm{eV}$, the As-adatom model is favored over the As-trimer. We find that clean Ga-terminated surfaces are favored with respect to the Asadatom surface only for very Ga-rich and As-poor conditions. For $\mu G a=\mu G a(b u l k)$ and $\mu A s=$ $\mu A s(b u l k)$, the As-adatom model is more stable than the Ga-adatom model by $1.5 \mathrm{eV} /(2 \times 2)$. Thus, stability of the As-adatom over the Ga-adatom surface requires $\mu_{A s}>\mu_{A s}$ (bulk) $-1.5 \mathrm{eV}$ [25]. Therefore, for Ga-rich conditions there is a $1 \mathrm{eV}$ wide window in chemical potential space $\mu A s(b u l k)-1.5$ $\mathrm{eV}<\mu_{A s}<\mu_{A s}$ (bulk) $-0.5 \mathrm{eV}$ where the As-adatom structure could be the most stable surface (For less Ga-rich conditions the window would be even wider.) We shall see below that for $\mathrm{T} \approx 700^{\circ} \mathrm{C}$ and an As pressure of $10^{-9}$ Torr, the As chemical potential does indeed lie within this window.

We calculate the As chemical potential for a gas of $\mathrm{As}_{2}$ molecules at pressure $\mathrm{p}$ and temperature T. Figure 8 shows the chemical potential for As as a function of temperature and pressure. The curves shown were computed using the standard expression[26]: 


$$
2(\mu A s-\mu A s(b u l k))=\text { Emole }-2 \mathrm{E} b u l k+k T \ln (p V Q / k T)-k T \ln \left(Z_{r o t}\right)-k T \ln \left(Z_{v i b}\right) .
$$

In this equation $\mathrm{Z}_{r o t}$ and $\mathrm{Z}_{v i b}$ are the rotational and vibrational partition functions for a molecule, $\mathrm{k}$ is Boltzmann's constant, $\mathrm{V}_{Q}$ is the quantum volume, Ebulk is the total energy per atom of bulk As at $\mathrm{T}=0 \mathrm{~K}$, and Emole is the energy of an $\mathrm{As}_{2}$ molecule at $\mathrm{T}=0 \mathrm{~K}$. Total energy calculations performed for $\mathrm{As}_{2}$ molecules and bulk As indicate that Emole - 2Ebulk $=2.4 \mathrm{eV}$. Using this result we find that for $\mathrm{T} \approx 700^{\circ} \mathrm{C}$ and $\mathrm{p}=10^{-9} \mathrm{Torr}, \mu_{A s}-\mu_{A s}(b u l k)=-1.2 \mathrm{eV}$. This chemical potential lies within the window required for stability of the As-adatom model, thereby providing support for our view that the $2 \times 2$ structure discussed above corresponds to the As adatom model.

Finally, these calculations predict that it is possible to stabilize the As-trimer structure in very As-rich conditions. As seen in Figure 5, equilibration of a $\mathrm{GaN}(0001)$ surface held at $\mathrm{T} \approx$ $500^{\circ} \mathrm{C}$ with an $\mathrm{As}_{2}$ gas at pressures greater than $\approx 10^{-6}$ Torr is expected to give rise to the As-trimer structure. However, these conditions have not been realized in the experiments discussed above.

\section{Conclusions}

In conclusion, we have studied the exposure of Ga-polar GaN(0001) surfaces to arsenic during growth. We find that the arsenic produces a strong $2 \times 2$ RHEED pattern which is otherwise not seen in clean $\mathrm{GaN}$ growth. A similar $2 \times 2$ pattern is observed by many workers during MBE-growth, and we believe that in many of these cases the growth systems used contain unintentional arsenic contamination thereby producing their $2 \times 2$ patterns. We have constructed a phase diagram for the Gapolar $\mathrm{GaN}$ (0001) surface exposed to arsenic, which displays the existence of a distinct $2 \times 2$ phase as well as a surfactant effect of arsenic. Based on Auger emission spectroscopy results we favor a simple As-adatom $2 \times 2$ model for this surface structure. We note that arsenic is not the only species which can produce a $2 \times 2$ reconstruction on $\mathrm{GaN}(0001)$; we have observed a $2 \times 2$ arrangement in the presence of $\mathrm{Mg}$ [21], and $2 \times 2$ patterns during growth with ammonia have been reported. Nevertheless, because of the similarity of our results with arsenic compared to those of other groups performing GaN MBE with a plasma-source for $\mathrm{N}$, we feel that the reports of $2 \times 2$ reconstructions in those cases do indeed arise from the presence of arsenic on the surface.

\section{Acknowledgements}

This work was supported by the Office of Naval Research, grant N00014-96-1-0214.

[11] A. R. Smith, R. M. Feenstra, D. W. Greve, M.-S. Shin, M. Skowronski, J. Neugebauer, and J. Northrup, Appl. Phys. Lett. 72, 2114 (1998).

[2] M. Seelmann-Eggebert, J. L. Weyher, H. Obloh, H. Zimmermann, A. Rar, and S. Porowski, Appl. Phys. Lett. 71, 2635 (1997).

[3] W. E. Packard, J. D. Dow, R. Nicolaides, K. Doverspike, and R. Kaplan, Superlattices and Microstructures 20, 145 (1996); J. Mater. Res. 12, 646 (1997).

[4] A. R. Smith, R. M. Feenstra, D. W. Greve, J. Neugebauer, J. E. Northrup, Phys. Rev. Lett. 79, 3934 (1997).

[5] A. R. Smith, R. M. Feenstra, D. W. Greve, M. S. Shin, M. Skowronski, J. Neugebauer and J. Northrup, Surf. Sci. 423, 70 (1999).

[6] R. Held, D. E. Crawford, A. M. Johnson, A. M. Dabiran, and P. I. Cohen, J. Electron. Mater. 26, 272 (1997); R. A. Held, G. Nowak, B. E. Ishaug, S. M. Seutter, A. Parkhomovsky, A. M. 
Dabiran, P. I. Cohen, I. Grzegory, and S. Porowski, J. Appl. Phys. 85, 7697 (1999).

[7] Y.-C. Chao, C. B. Stagarescu, J. E. Downes, P. Ryan, K. E. Smith, D. Hanser, M. D. Bremser, and R. F. Davis, Phys. Rev. B 59, R15586 (1999).

[8] V. M. Bermudez, D. D. Koleske, and A. E. Wickenden, Appl. Surf. Sci. 126, 69 (1998).

[9] A. R. Smith, R. M. Feenstra, D. W. Greve, J. Neugebauer, and J. Northrup, Appl. Phys. A 66, S947 (1998).

[10] A. R. Smith, R. M. Feenstra, D. W. Greve, M. S. Shin, M. Skowronski, J. Neugebauer, and J. Northrup, J. Vac. Sci. Technol. B 16, 2242 (1998).

[11] Q. K. Xue, Q. Z. Xue, R. Z. Bakhtizin, Y. Hasegawa, I. S. T. Tsong, T. Sakurai, and T. Ohno, Phys. Rev. Lett. 82, 3074 (1999).

[12] K. Iwata, H. Asahi, S. J. Yu, K. Asami, H. Fujita, M. Fushida, and S. Gonda, Jpn. J. Appl. Phys. 35, L289 (1996).

[13] P. Hacke, G. Feuillet, H. Okumura, and S. Yoshida, Appl. Phys. Lett. 69, 2507 (1996).

[14] A. R. Smith et al., MRS Internet J. Nitride Semicond. Res. 3, 12 (1998), and references therein.

[15] E. S. Hellman, MRS Internet J. Nitride Semicond. Res. 3, 11 (1998).

[16] E. J. Tarsa, B. Heying, X. H. Wu, P. Fini, S. P. DenBaars, and J. S. Speck, J. Appl. Phys. 82, 5472 (1997).

[17] V. Ramachandran, A. R. Smith, R. M. Feenstra and D. W. Greve, J. Vac. Sci. Technol. A 17, 1289 (1999).

[18] T. Zywietz, J. Neugebauer, and M. Scheffler, Appl. Phys. Lett. 73, 487 (1998).

[19] Y. Zhao, F. Deng, S. S. Lau, and C. W. Tu, J. Vac. Sci. Technol. 16, 1297 (1998).

[20] T. Zywietz, J. Neugebauer, M. Scheffler, J. Northrup, and C. G. Van de Walle, MRS Internet J. Nitride Semicond. Res. 3, 26 (1998).

[21] V. Ramachandran, R. M. Feenstra, W. L. Sarney, L. Salamanca-Riba, J. E. Northrup, L. T. Romano, and D. W. Greve, Appl. Phys. Lett. 75, xxx (1999), to appear.

[22] J. E. Northrup and J. Neugebauer, Phys. Rev B 53, 10477 (1996).

[23] J. Neugebauer, T. K. Zywietz, M. Scheffler, J. E. Northrup and C. G. Van de Walle Phys. Rev. Lett. 80, 3097 (1998).

[24] J. E. Northrup, R. Di Felice, and J. Neugebauer, Phys. Rev. B 56, 4325 (1997).

[25] In the Ga-rich limit, the experimentally observed reconstruction is a pseudo-1x1 structure and not the simple $2 \times 2$ Ga-adatom. However, the surface energy of the pseudo- $1 \times 1$, which is thought to be a laterally contracted double-layer of Ga atoms, is estimated to be $<0.2 \mathrm{eV} / 2 \times 2$ lower than the simple Ga-adatom model (see Ref. [10]). This difference in surface energy does not effect the qualitative conclusions drawn here.

[26] C. Kittell, Thermal Physics (Wiley, New York, 1969). 

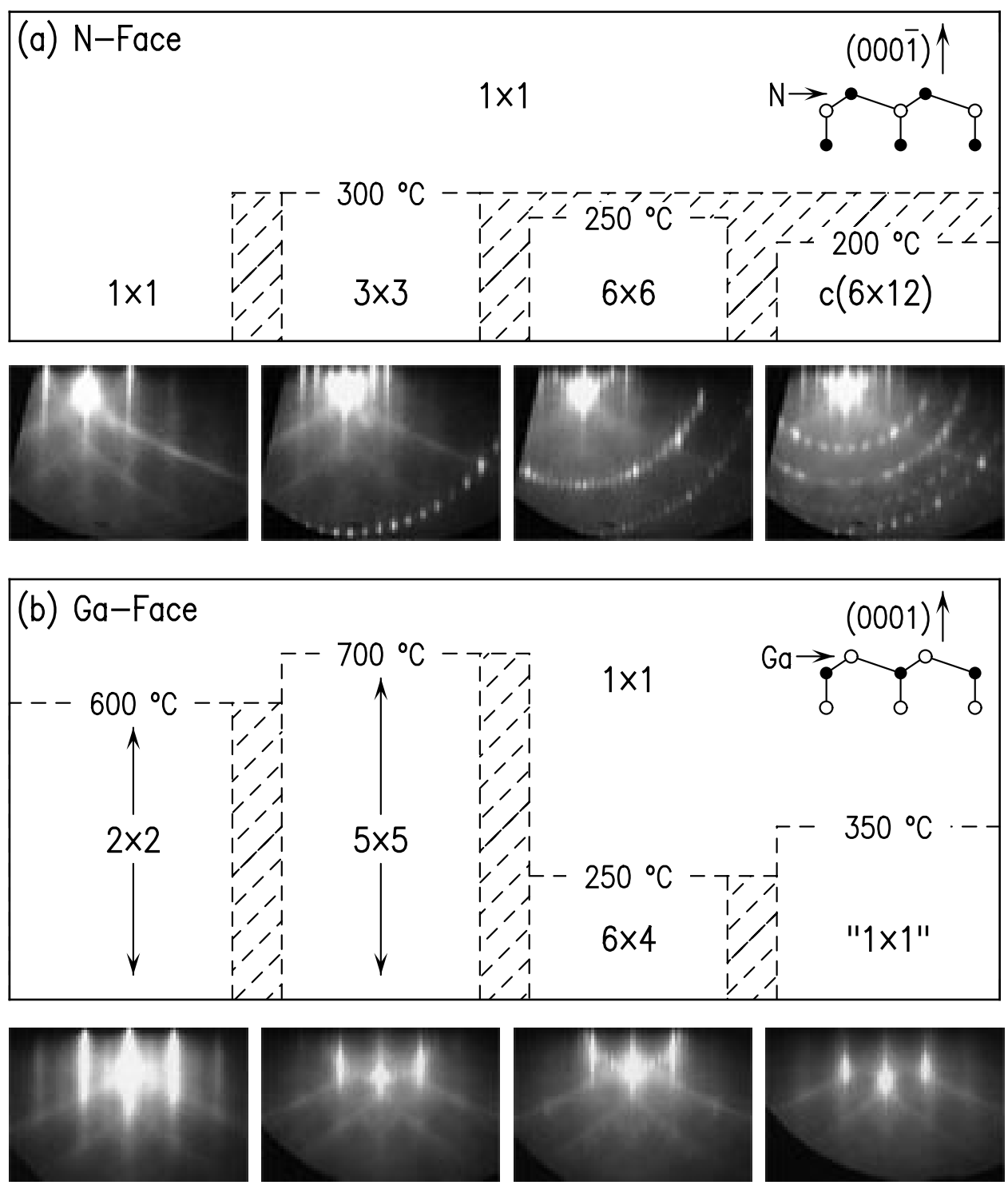

$\mathrm{N}-$ Rich

Ga-Rich

Figure 1 Schematic phase diagrams illustrating the coverage and temperature dependence of the reconstructions existing on the (a) (000 $\overline{1})$ surface, and (b) (0001) surface. Ga coverage increases from left to right in both diagrams. Temperatures given correspond to either order-disorder phase transitions or annealing transitions. Cross-hatched regions indicate either mixed or intermediate phases. RHEED patterns for both surfaces, as viewed along the [112 0$]$ azimuth, are also shown. 


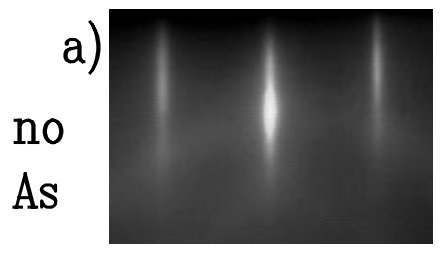

b)
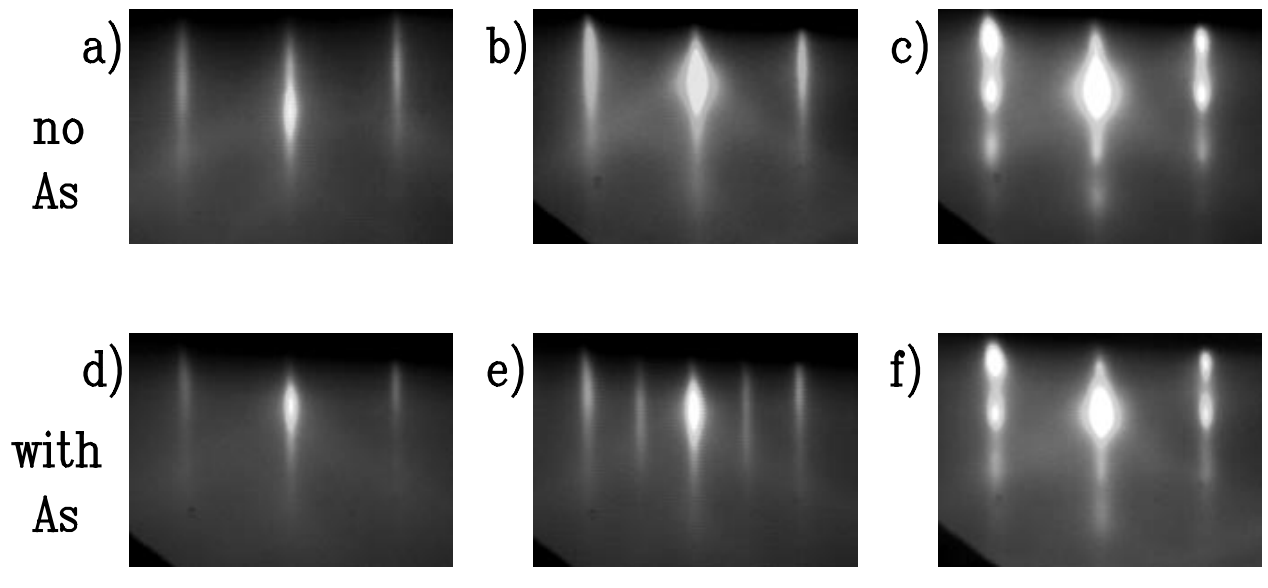

e)
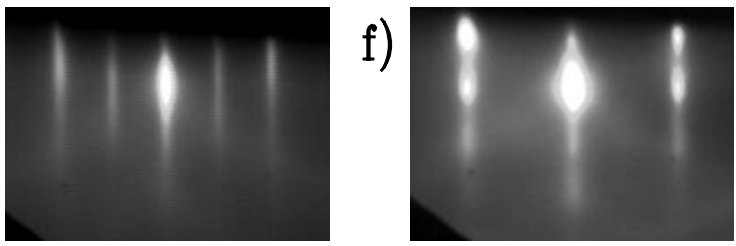

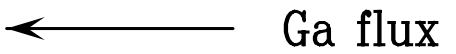

Figure 2 (a)-(c) RHEED patterns of Ga-polar films during growth without any arsenic, as a function of decreasing Ga flux. (d)-(f) A similar progression of RHEED patterns in the presence of arsenic with a BEP of $1 \times 10^{-9}$ Torr. 

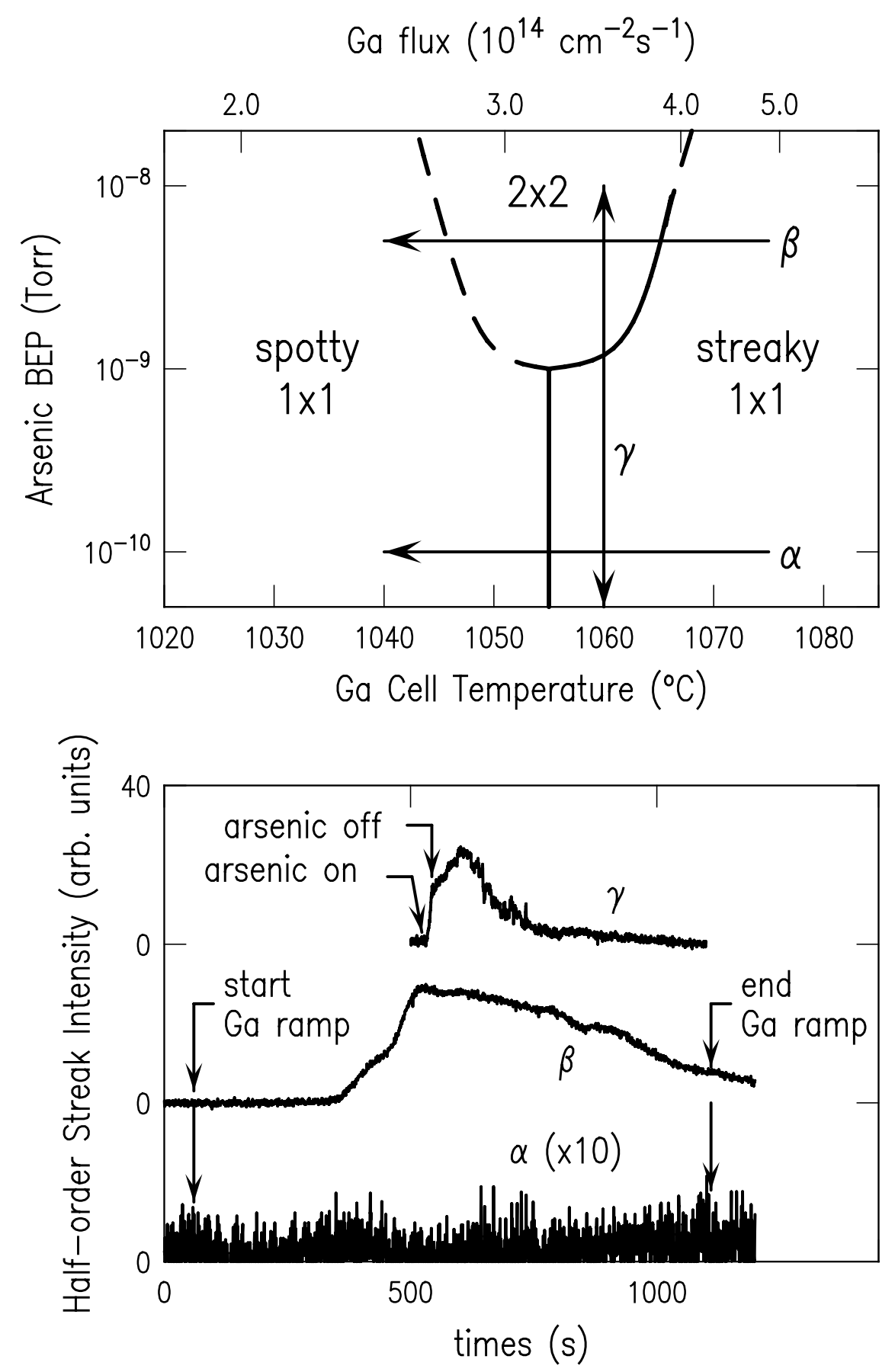

Figure 3 (a) Different phases of the Ga-polar GaN surface under varying Ga and As flux conditions. (b)Intensity of half order RHEED streaks as a function of time: $(\alpha)$ Ga flux sweep with a low arsenic flux, $(\beta)$ Ga flux sweep with arsenic BEP about $1 \times 10^{-9}$ Torr and $(\gamma)$ turning arsenic on and off. 


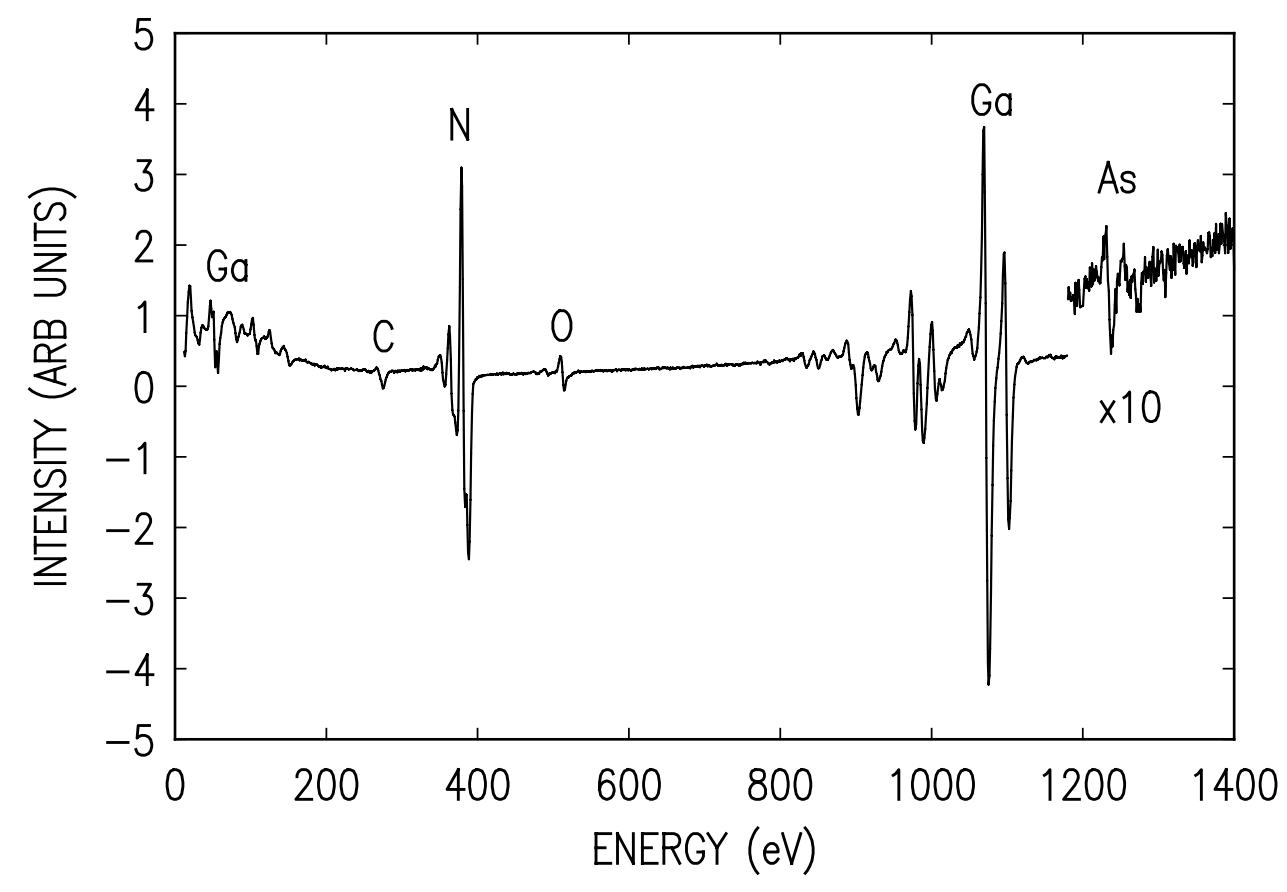

Figure 4 Auger emission spectrum obtained from the $2 \times 2 \mathrm{GaN}(0001)$ surface, with incident electron energy of $3 \mathrm{keV}$.

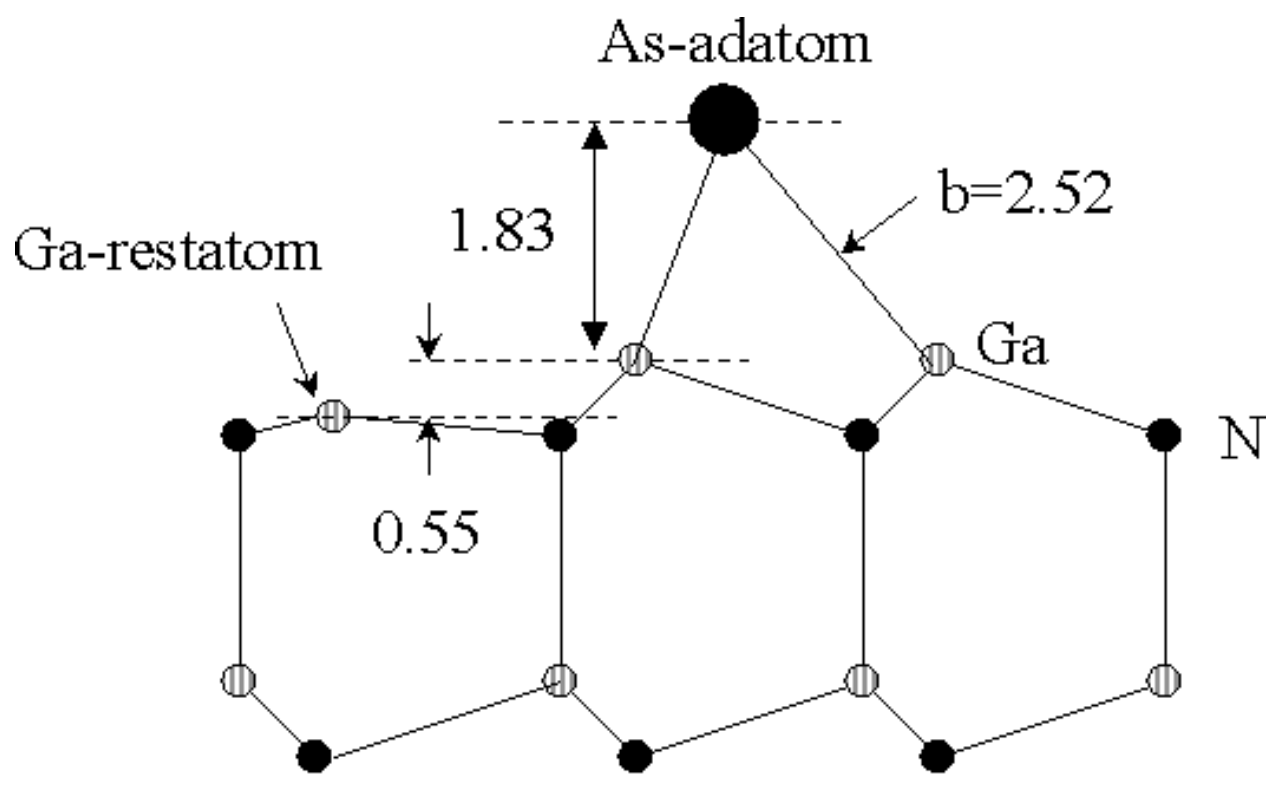

As-adatom in $\mathrm{H} 3$ site on $\mathrm{GaN}(0001) 2 \times 2$

Figure 5 Schematic model of arsenic adatom in $\mathrm{H} 3$ site. All dimensions are given in angstroms. 


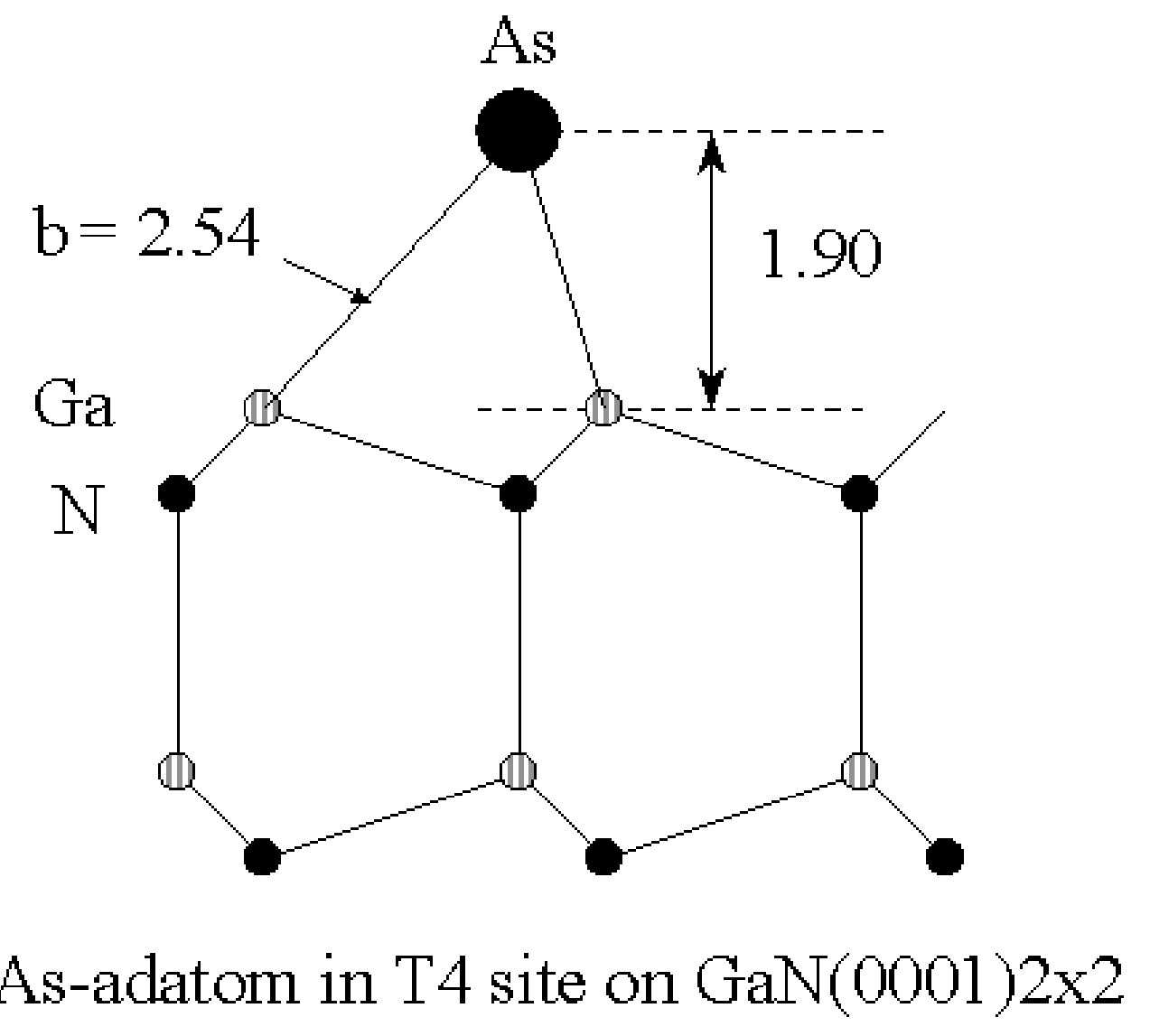

Figure 6 Schematic model of arsenic adatom in T4 site. All dimensions are given in angstroms. 


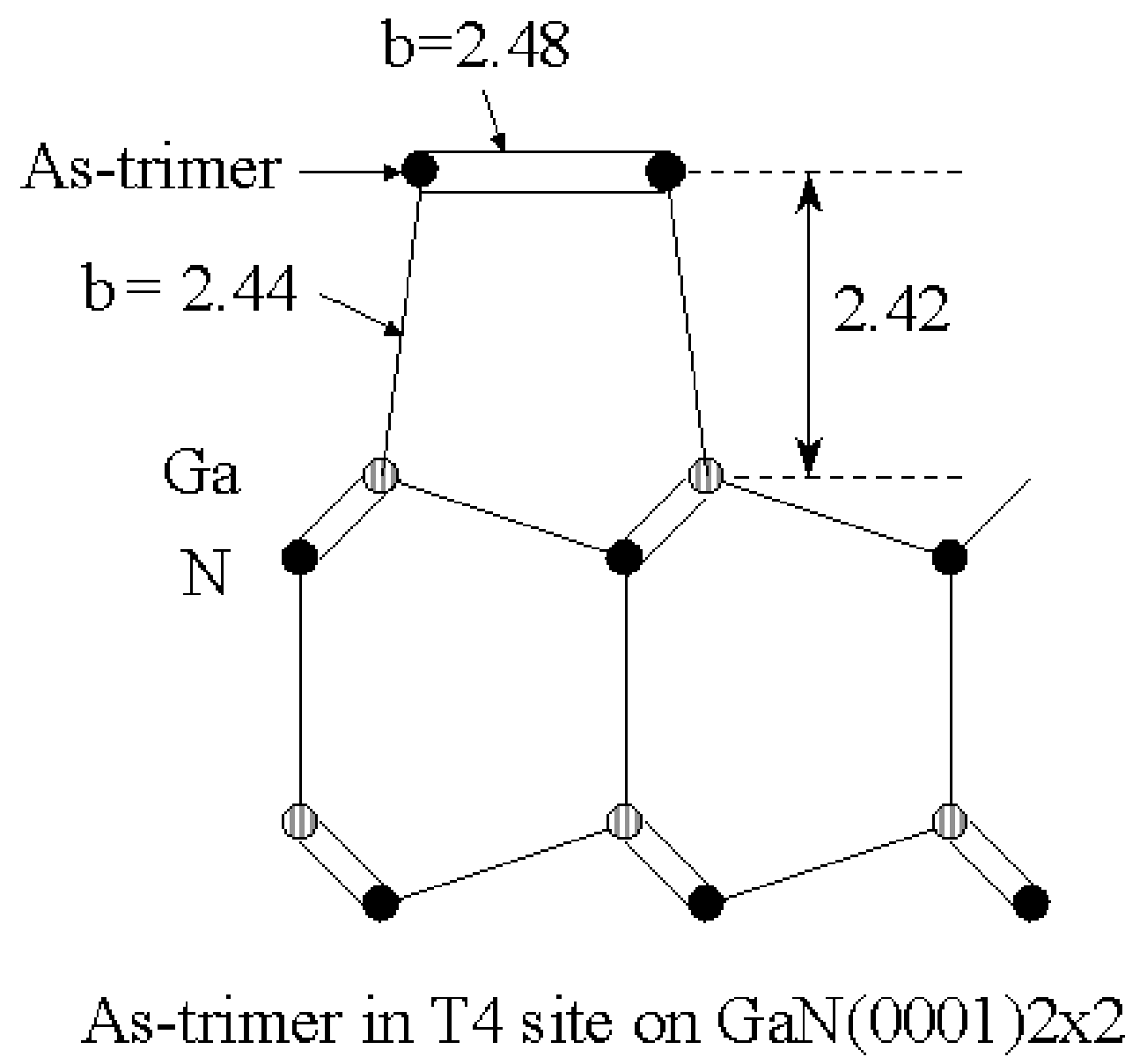

Figure 7 Schematic model of arsenic trimer in T4 site. All dimensions are given in angstroms. 


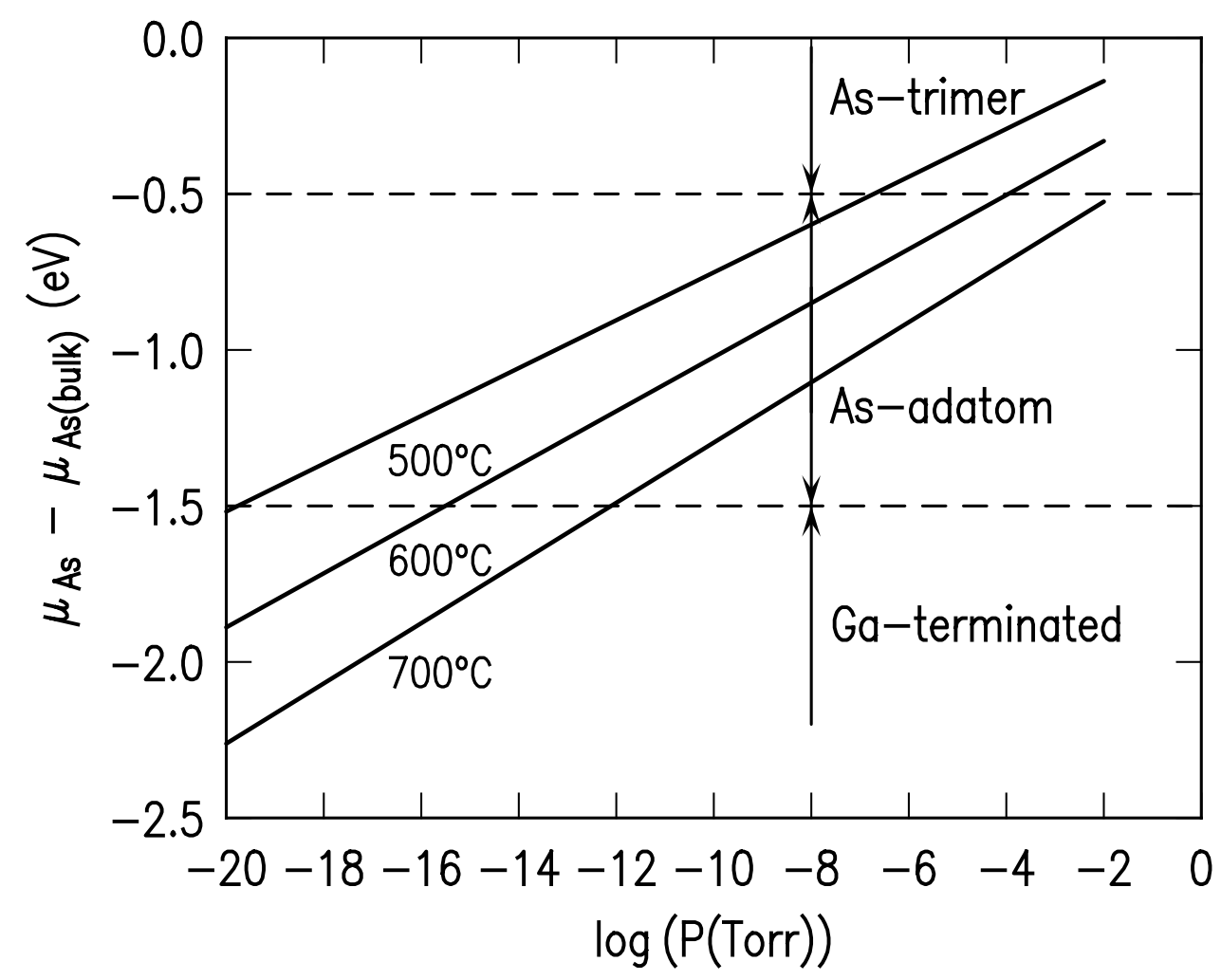

Figure 8 Plot of the chemical potential of As as a function of pressure for three different values of the temperature $\mathrm{T}$. The chemical potential varies with pressure as $\mathrm{kT} \ln (\mathrm{pV} Q / \mathrm{kT})$. The horizontal dashed lines correspond to values of the As chemical potential where transitions between stable surface structures are expected based on our first principles calculations. See the text for further details. 